\title{
3D printing for the planning and use in complicated surgical procedures: an interview with Juling Ong
}

\author{
Juling Ong ${ }^{*, 1}$ \\ ${ }^{1}$ Great Ormond Street Hospital for Children NHS Foundation Trust Great Ormond Street London WC1N 3JH \\ *Author for correspondence: juling.ong@gosh.nhs.uk
}

First draft submitted: 23 September 2019; Published online: 18 December 2019

Keywords: 3D models $\bullet$ pediatrics $\bullet$ 3D printing $\bullet$ anatomy $\bullet$ craniofacial $\bullet$ surgery

Juling Ong is a Consultant Craniofacial and Plastic Surgeon at Great Ormond Street Hospital (London, UK) working with the craniofacial team. His role as the head of the $3 \mathrm{D}$ printing department is to develop the service and make it useful for every hospital teams including clinical services and research groups. He hopes he can help facilitate the work that they do in developing innovative treatments for children with rare diseases and complex medical problems.

This interview was conducted by Georgi Makin, Editor of 3DMedNet, a sister site to the Journal of $3 D$ Printing in Medicine.

\section{What led you to your current focus?}

During my fellowship in Austin (TX, USA), there was a lot of work taking place using 3D planning for orthognathic and craniofacial surgery. I wanted to bring that interest back to the UK. I believe it can offer a lot of benefits, especially with the more complex cases. 3D technology provides a significant amount of additional information that is useful in reducing risk and reducing operation time. We are also able to achieve our outcomes with much better definition, with the assurance that we are getting the best accuracy possible.

When it comes to pediatrics, particularly in hospitals such as Great Ormond Street Hospital that deal with very rare conditions, there are often very few previous case examples worldwide from which we can learn from. When we see children with unique conditions for the first time, $3 \mathrm{D}$ technology gives us more information to work with. This information is useful because it allows us to learn more about unique anatomy, helps us to identify and reduce the operating risks and then allows us to plan different strategies in relation to their unique situation. This may involve making 3D models or using virtual models, which really helps to facilitate discussions from different members of the medical team: surgeons, nurses, radiologists etc., all from different backgrounds. Being able to picture the problem by holding the model in your hand is a very helpful way of looking at it. These models are invaluable tools which allow people from different backgrounds to understand the situation, as a team.

\section{Can you tell me about the general workflow involved when manufacturing 3D printed models for clinical purposes?}

We will normally first receive a request from a clinician, about a new patient. We will want to know more about the condition, anatomy and the clinical options that are available. In addition, we need to analyze the patient's three-dimensional cross-sectional data, such as from CT or MRI scans captured in the hospital. The information is then transferred to our department's 3D medical software that allows us to segment the scan and produce a 3D model of the anatomy. Depending on the remit of the project or the clinical problem, we will go through various treatment options with the clinician or surgeon, and then often develop a few different strategies, looking for the best option for that patient. The virtual planning happens on the screen and often with the physical models.

In a recent case involving the successful separation of conjoined twins, Safa and Marwa, we utilized a wide range of $3 \mathrm{D}$ technology in order to analyze the problem surrounding the unique anatomy of the two girls. The model we wanted to produce was for a very rare condition, found in only one in 2,000,000 births, meaning our experience of similar cases was very limited. The twins' brains were quite deformed due to where they were conjoined, and 
from their brain models, we could see that their brains were completely intertwined with each other. We therefore produced numerous models of the patient before they had surgery. We were then able to physically cut the models to trial several potential approaches and procedures. The models were designed to familiarize the surgical team with the size of the skulls, where their suture lines were and the location of the relevant anatomy on the bone surface and of the brain, dura and vasculature.

\section{Could you tell me more about how the models were used in this case to inform the surgical plan?}

The models allowed us to test how the twins could be separated by various treatment strategies. We could then identify the best surgical plan available and work towards the best possible outcome. In this case, we were aiming to create symmetrical skulls with a minimal time in the operating theatre, minimise bleeding and using as little hardware as possible in the patients. It was also vital to be able to understand how best to maintain an adequate blood supply and blood drainage to the two brains, after separations, so understanding the anatomy of the blood vessels was key.

We spent a lot of time analyzing their MRIs and computed tomography angiograms, and then segmented them to map out the venous drainage and arterial supply to the two brains. We identified that they shared a common ring sinus, which is a feature in total vertical craniopagus twins however, interestingly, we found that there was a complex triangular rearrangement of veins, which we would need to negotiate in order to separate them safely. Furthermore, their brains shared arterial circulation in certain areas, which presented a significant challenge in terms of determining how to safely divide the arteries between the twins in order to proceed with the separation.

\section{How was 3D printing technology used to assist you during the operation?}

We designed skin flaps based on our previous experience with other conjoined twins. Underlying these flaps, skull osteotomies were added which helped us identify where to make surgical cuts. Surgical guides were produced and on the surface of these, we mapped out underlying structures of interest, such as the venous anatomy, with color. An additional guide was made to show the location of the blood vessels that crossed from one brain to the other. This helped show where the separation of the brains needed to take place and allowed us to easily identify the location of the arteries during the operation.

To produce the preliminary models, we used a 3D printer which can print in different colors and textures, allowing us to differentiate the different anatomies as clearly as possible. Using clear plastics made from flexible, rigid and colored materials, arteries and veins within the brain could be clearly identified. The guides for the surgery made using a material that was suitable for use in the operative environment, but also allowed for the demonstration of the anatomy.

\section{How do you see the field of 3D printing growing into the future?}

The field of 3D printing is growing at a rapid rate, particularly in healthcare and I am sure will only continue to grow. I believe that it will become a more acceptable and even essential part of everyday healthcare, not just forthe few referral centers. I expect that every healthcare provider will have some access to $3 \mathrm{D}$ technology in the near future. Additionally, I envision the technology being increasingly utilized in analysis, and in the near future it will be the method by which we record our pre-operative decisions along with operative, surgical and treatment outcomes.

With continual development in the materials, technology and printers we use, 3D printing in medicine will become increasingly complex and sophisticated. Developments in materials, will provide us with implants that function better and more reliably in the human body. This technology will be increasingly integrated with advances in artificial intelligence and machine learning. Augmented and virtual reality environments will make use of 3D information combined with clinical information, in order to aid surgeons and physicians in decision-making and advanced treatment options.

\section{What advice would you give to someone considering setting up their own 3D printing facility?}

There are number of challenges a 3D printing facility faces, the first being the cost. It is expensive to set up a functional facility, which is why I was very grateful to have the help of the Great Ormond Street Children's Charity (London, UK) which funded our project and the Biomedical Research Centre (London, UK) for supporting 
this project. Furthermore, if you are going to set up a 3D service in a hospital, you are likely to benefit from the engagement and involvement of as many different specialties and departments as possible. We continue to learn from other departments, with each of them bringing different knowledge and innovations which can benefit other applications. I have always strived to ensure that the service we provide is open to all specialties, which will hopefully result in increasing understanding about the various benefits of $3 \mathrm{D}$ technology in medicine. I think the most important part of your 3D facility is the level of expertise. Having a dedicated engineer/technician that is completely focused on supporting the service is vital.

In conclusion, starting a new 3D service at your hospital, it is likely to stimulate a great deal of interest and help to get people excited about the potential of 3D technology. From my point of view, 3D printing models help to reduce risk by helping surgeons to understand the unique anatomies of the patients in greater detail. Other advantages include using the models to educate the patients about their anatomy and/or the surgical plan and an improved and more informed consent process for these complex rare patients.

\section{Financial Disclosure}

Juling Ong has no relevant affiliations or financial involvement with any organization or entity with a financial interest in or financial conflict with the subject matter or materials discussed in the manuscript. This includes employment, consultancies, honoraria, stock ownership or options, expert testimony, grants or patents received or pending, or royalties.

No writing assistance was utilized in the production of this manuscript.

Interview Disclosure

The opinions expressed in this interview are those of Juling Ong and do not necessarily reflect the views of Future Medicine Ltd. 